\title{
РАСПРЕДЕЛЕНИЕ БОЛЬЦМАНА В ЗАДАЧЕ МИГРАЦИИ НАСЕЛЕНИЯ
}

\author{
(c) 2020 И. В. Данилова ${ }^{\bowtie}$, А. Н. Кириллов, А. А. Крижановский \\ Институт прикладных математических исследований \\ Карельского научного иентра Российской академии наук \\ ул. Пушкинская, 11, 185910 Петрозаводск, Российская Федераиия
}

\begin{abstract}
Аннотация. В данной работе рассматривается задача миграции населения РФ между тремя группами федеральных округов. В первую группу вошли Центральный и Северо-Западный, во вторую - Дальневосточный, Уральский и Сибирский, в третью - Южный, Приволжский и Северо-Кавказский федеральные округа. Предложена модель динамики численностей населения с учетом распределения Больцмана, которое описывает распределение мигрирующего населения по трем заданным территориям. Предложена функция полезности, входящая в распределение Больцмана и, так же предложена, входящая в функцию полезности, мера информированности (притягательности) населения по отношению к рассматриваемым территориям. При этом, мера информированности учитывает экономический фактор - долю населения, живущего выше прожиточного минимума и общую численность населения на рассматриваемых группах федеральных округов. Предполагается, что чем больше общая численность населения на данной территории, тем больше информации об этой территории имеется. Проводится идентификация параметров, характеризующих коэффициенты естественного прироста и доли мигрирующего населения рассматриваемых территорий при различных значениях параметра, характеризующего оптимальность распределения населения по трем заданным территориям. Идентификация параметров модели реализована с помощью метода градиентного спуска с дроблением шага на языке программирования Рython3.5. Так же для реализации идентификации параметров использовались данные из официальных статистических источников. На основе проведенной идентификации получены значения распределения Больцмана и миграционных потоков между тремя группами федеральных округов, проведено сравнение модельных данных с реальными, построен прогноз численностей населения для каждой территории. Показано, что значение параметра оптимальности влияет на прогноз численности населения всех трех групп федеральных округов.

Ключевые слова: миграция населения, распределение Больцмана, функция полезности, мера информированности, идентификация параметров.
\end{abstract}

\section{ВВЕДЕНИЕ}

Моделирование миграционных процессов играет важную роль в анализе и прогнозировании их влияния на уровень жизни населения в регионах. Один из подходов к моделированию миграционных процессов заключается в описании взаимодействия между рассматриваемыми территориями через закон всемирного тяготения $[1,2]$. Еще один подход

Данилова Инна Владимировна e-mail: DanilovaInna1987@mail.ru заключается в построении вероятностных моделей миграционных процессов. В работе [3] рассматриваются этапы построения общей модели межрегиональной миграции с учетом вероятности перехода субпопуляции (части населения, имеющей некоторый общий признак - культура, этническое или социальное происхождение) из региона $i$ в регион $j$ в единицу времени, при этом не учитывается циркуляция мигрантов внутри одного региона и также не учитывается естественный прирост населения. Вероятности перехода из одного состояния в другое в еди-

(†) Контент доступен под лицензией Creative Commons Attribution 4.0 License. The content is available under Creative Commons Attribution 4.0 License. 
ницу времени представлены в экспоненциальной форме и зависят от функций полезности $i$-го и $j$-го регионов. Функции полезности зависят от характеристик регионов, задаваемых членами субпопуляции. Сами же характеристики не конкретизируются.

Третий подход предлагает теория оптимального фуражирования, согласно которой, популяция или отдельная особь действует таким образом, чтобы максимизировать скорость потребления энергии $[4,5]$. Выбор популяцией пригодного для жизни ареала (местообитания) является одной из основных задач. В работе [6] рассматривается теоретико-игровой подход в задаче оптимального поведения взаимодействующих популяций типа «хищник-жертва», находящихся в некотором ареале с учетом миграции из него и внутривидовой конкуренции, показано, что система, описывающая взаимодействие двух популяций, с течением времени выходит на стационарный режим. В работе [7] рассматривается задача выбора популяцией ареала, при условии, что популяция, находясь в одном из них, оценивает все остальные [7]. Подход к решению этой задачи заключается в построении функции полезности $U_{i j}$, оцениваемого $j$-го ареала, популяцией, находящейся в $i$-м ареале. При этом, вероятность выбора популяцией ареала задается распределением Больцмана $p_{i j}=e^{q U_{i j}} / \sum_{j=1}^{m} \exp \left(q U_{i j}\right)$, где $m$ - количество ареалов, $i, j=1, \ldots, m q-$ положительный параметр, отвечающий за оптимальность распределения популяции по ареалам. Если значение параметра $q$ достаточно мало, то популяция будет распределяться среди ареалов случайным образом, т. е. $p_{i j}=1 / m$; если $q$ достаточно велико, то популяция с большей вероятностью выберет ареал с большим значением функции полезности $U_{i j}$. В работе [7] функция полезности имеет следующий вид: $U_{i j}=V_{j} I_{i j}+\left(1-I_{i j}\right) \bar{V}-T_{i j}, i, j=1, \ldots, m$, где $I_{i j}-$ мера информированности популяции, находящейся в ареале $i$ об ареале $j, V_{j}-$ объем ресурсов питания в ареале $j, \bar{V}-$ средняя полезность ареалов, $T_{i j}$ - функция затрат на перемещение из $i$ в $j$. В работе [8] доказана устойчивость распределения Больцмана для двух ареалов.
Чтобы конкретизировать меру информированности, нужно определить носители информации и их взаимодействие с окружающей средой. В [9] рассматривается эконофизическая концепция информационного взаимодействия между носителями информации. В работах [10-16] исследуются вопросы принятия решения популяцией о выборе стратегии с учетом накопления и потери данных с течением времени и показано, что стратегия поиска и переход между ними зависят от роста информированности.

В данной работе предлагается модель, описывающая динамику численности населения на территории Российской Федерации на основе подхода [7], с учетом меры информированности, входящей в распределение Больцмана. При этом не учитывается отток населения за границу и приток из-за границы. Проведена идентификация параметров модели, характеризующих естественный прирост и миграцию населения в каждой группе федеральных округов при различных значениях $q$.

На основе результатов идентификации проводится анализ миграционных потоков между тремя группами федеральных округов и сравнение модельных данных со статистическими, строится прогноз численностей населения для каждой группы.

\section{1. ПОСТАНОВКА ЗАДАЧИ}

\section{1. Модель динамики численности населения в трех группах федеральных округов РФ}

В данной работе территория РФ делится на три группы федеральных округов. В первую группу (территория 1) входят Центральный и Северо-Западный федеральные округа, во вторую (территория 2) - Дальневосточный, Уральский и Сибирский, в третью (территория 3) - Южный, Приволжский и Северо-Кавказский. Предполагается, что мигрирующее население распределяется по трем группам федеральных округов согласно распределению Больцмана.

Рассмотрим модель, описывающую динамику численности населения для каждой из трех заданных территорий: 


$$
\left\{\begin{array}{l}
\dot{x}_{1}=k_{1}\left(1-\gamma_{1}\left(1-p_{1}\right)\right) x_{1}- \\
-\gamma_{1} p_{2} x_{1}-\gamma_{1} p_{3} x_{1}+\gamma_{2} p_{1} x_{2}+\gamma_{3} p_{1} x_{3}, \\
\dot{x}_{2}=k_{2}\left(1-\gamma_{2}\left(1-p_{2}\right)\right) x_{2}- \\
-\gamma_{2} p_{1} x_{2}-\gamma_{2} p_{3} x_{2}+\gamma_{1} p_{2} x_{1}+\gamma_{3} p_{2} x_{3}, \\
\dot{x}_{3}=k_{3}\left(1-\gamma_{3}\left(1-p_{3}\right)\right) x_{3}- \\
-\gamma_{3} p_{1} x_{3}-\gamma_{3} p_{2} x_{3}+\gamma_{1} p_{3} x_{1}+\gamma_{2} p_{3} x_{2},
\end{array}\right.
$$

где $x_{i}$ - численность населения $i$-й территории, $k_{i}$ - коэффициент естественного прироста населения $i$-й территории, $\gamma_{i}-$ доля населения $i$-й территории, включающая в себя население, мигрирующее внутри собственной территории и за ее пределы, $p_{i}$ вероятность выбора $i$-й территории мигрирующим населением, $i=1,2,3, p_{1}+p_{2}+p_{3}=1$. Тогда $\gamma_{i j}=\gamma_{i} p_{j}$ - доля мигрирующего населения территории $i$, выбравшая территорию $j$, где $i, j=1,2,3$. Опираясь на подход [7], вероятность перехода на территорию $j$ будем описывать распределением Больцмана: $p_{j}=\exp \left(q U_{j}\right) / \sum_{k=1}^{3} \exp \left(q U_{k}\right), \quad j, k=1,2,3$, где $U_{j}=V_{j} I_{j}-$ полезность $j$-й территории с точки зрения мигрирующей части населения, $V_{j}-$ истинная полезность территории $j$. Под истинной полезностью будем понимать среднее значение индекса дохода, являющегося одной из трех компонент индекса человеческого развития (ИЧР) $[16,18], I_{j}=\beta_{j} x_{j} /\left(\beta_{j} x_{j}+c_{j}\right)-$ мера притягательности территории $j, \beta_{j}-$ доля населения территории $j$, доход которого выше прожиточного минимума, $c_{j}-$ константа, характеризующая накопленную в среднем информацию о территории $j$, будем считать, что носителем информации является все население данной территории.

\section{2. МАТЕРИАЛЫ И МЕТОДЫ ИССЛЕДОВАНИЯ}

\section{1. Идентификация параметров модели}

Для системы (1) проводится идентификация набора параметров $S=\left(\gamma_{1}, \gamma_{2}, \gamma_{3}, k_{1}, k_{2}, k_{3}\right)$ c помощью метода градиентного спуска с дроблением шага, т. е. численно решается задача минимизации функции

$$
\begin{gathered}
F(S)=\delta_{1} \sum_{r=1}^{n}\left(x_{1 r}-x_{1 r}^{*}(t, S)\right)^{2}+ \\
+\delta_{2} \sum_{r=1}^{n}\left(x_{2 r}-x_{2 r}^{*}(t, S)\right)^{2}+\delta_{3} \sum_{r=1}^{n}\left(x_{3 r}-x_{3 r}^{*}(t, S)\right)^{2}
\end{gathered}
$$

по набору параметров $S$, где $x_{i r}$ - реальные значения численности населения в моменты времени $t_{r}$, а $x_{i r}^{*}$ - решения системы (1), найденные методом Рунге - Кутты. В качестве значений масштабирующих параметров $\delta_{i}$, $i=1,2,3$, берутся средние доли численностей населения в каждой из трех групп федеральных округов РФ.

Для определения реальных значений численностей населения использовались данные с официального сайта Федеральной службы государственной статистики [17] за 19982014 гг. Параметры $V_{i}, \beta_{i}, c_{i}$ являются постоянными параметрами системы (1). Для вычисления индекса дохода $V_{i}$ в отдельном федеральном округе использовались данные о валовом региональном продукте (ВРП) для субъектов РФ $[17,19]$ и курсе валют [20] в РФ за 1998-2014 гг. Из расчетов на основе статистических данных были рассчитаны постоянные параметры модели (1): $V_{1}=0,6013$, $V_{2}=0,572, V_{3}=0,498, \beta_{1}=0,81, \beta_{2}=\beta_{3}=0,78$, в качестве значения $c_{i}$ берется средняя численность населения на территории $i$ за 19982014 гг. Начальные значения идентифицируемых параметров определяются следующим образом: $k_{i}-$ среднее значение коэффициента естественного прироста, $\gamma_{i}$ - среднее значение мигрирующего населения территории $i$ за 1998-2014 гг. Метод идентификации параметров реализован на языке Python 3.5 [21]. Результаты представлены в табл. 1.

\section{3. ОБСУЖДЕНИЕ РЕЗУЛЬТАТОВ}

На основе результатов идентификации параметров $S$, получены значения вероятностей $p_{i}$ (см. рис. 1) и миграционных потоков между тремя группами федеральных округов $\gamma_{i j}$ (рис. 2). На рис. 1 и 2 в качестве $p_{i}$ и $\gamma_{i j}$ берутся средние значения распределения Больцмана и миграционных потоков за 1998-2014 гг. при некотором значении параметра q. На рис. 2 приведены результаты анализа иденти- 
Таблииа 1. Результаты идентификации параметров при заданных значениях $q$

[Table 1. Results of the identification of parameter for given values of $q$ ]

\begin{tabular}{|c|c|c|c|c|c|c|}
\hline$q$ & $\gamma_{1}$ & $\gamma_{2}$ & $\gamma_{3}$ & $k_{1}$ & $k_{2}$ & $k_{3}$ \\
\hline 0 & 0,012343 & 0,023900 & 0,015522 & $-0,004210$ & $-0,002100$ & $-0,000890$ \\
\hline 0,1 & 0,012360 & 0,023902 & 0,015501 & $-0,004227$ & $-0,002108$ & $-0,000868$ \\
\hline 1 & 0,012516 & 0,023918 & 0,015308 & $-0,004380$ & $-0,002172$ & $-0,000675$ \\
\hline 5 & 0,013166 & 0,023894 & 0,014443 & $-0,005055$ & $-0,002412$ & 0,000150 \\
\hline 10 & 0,013853 & 0,023682 & 0,013360 & $-0,005890$ & $-0,002576$ & 0,001076 \\
\hline 20 & 0,014805 & 0,022756 & 0,011357 & $-0,007426$ & $-0,002533$ & 0,002532 \\
\hline 30 & 0,015127 & 0,021356 & 0,009669 & $-0,008698$ & $-0,002088$ & 0,003450 \\
\hline 50 & 0,013337 & 0,017885 & 0,006891 & $-0,010291$ & $-0,000294$ & 0,003720 \\
\hline 70 & 0,014389 & 0,016807 & 0,006345 & $-0,011838$ & 0,001126 & 0,004197 \\
\hline 90 & 0,014442 & 0,015391 & 0,005834 & $-0,012658$ & 0,002348 & 0,004119 \\
\hline 100 & 0,014341 & 0,014725 & 0,005650 & $-0,012921$ & 0,002824 & 0,004037 \\
\hline
\end{tabular}

фикации параметров, характеризующих долю мигрирующего населения территории $i$. С помощью предложенной модели оценим рациональность выбора территории $i$.

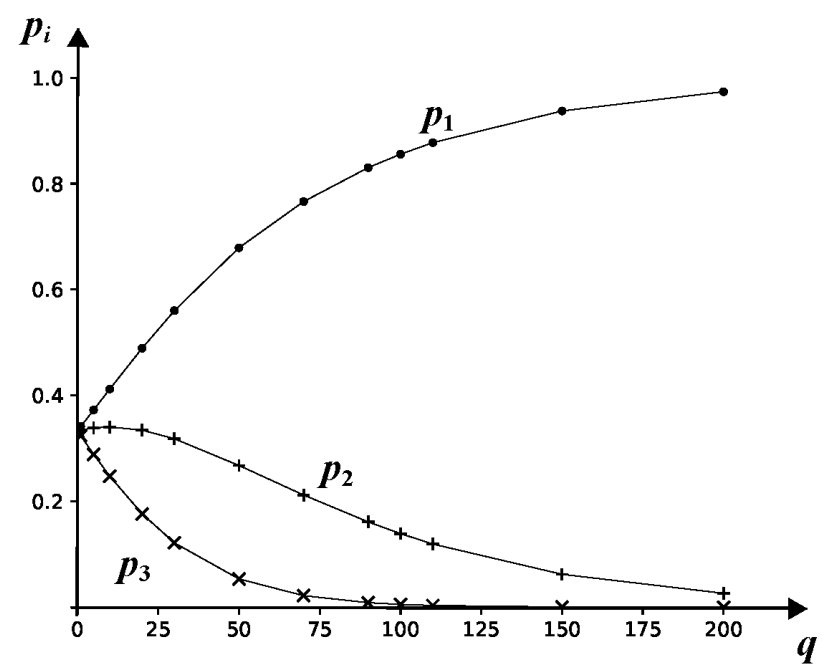

Рис. 1. Распределение Больимана при различных значениях $q$

[Fig. 1. Migration from the 1st, 2nd, and 3rd groups of the FD q]

На рис. 1 видно, что первый регион, согласно предлагаемой модели, наиболее предпочтителен для мигрирующей части населения РФ: $p_{1}>p_{2}>p_{3}$. Следовательно, территория 1 имеет самое высокое значение функции полезности $U_{1}>U_{2}>U_{3}$. Это справедливо, поскольку, согласно расчетам, на территории 1 самый высокий индекс дохода $V_{1}>V_{2}>V_{3}$ и самый высокий уровень прожиточного минимума $\beta_{1}>\beta_{2}=\beta_{3}$.

Из табл. 1 следует, что при различных значениях параметра q население второй группы регионов самое подвижное, при $q<10$ население первой группы самое инертное: $\gamma_{2}>\gamma_{3}>\gamma_{1}$, при $q>10$ самым инертным будет население третьей группы: $\gamma_{2}>\gamma_{1}>\gamma_{3}$. При этом, с ростом параметра $q$ подвижность населения территорий 2 и 3 снижается, а подвижность населения территории 1 меняется.

Рис. 2 иллюстрирует, что для мигрирующего населения территорий 1 и 2 наименее привлекательна территория 3. Это так же можно объяснить тем, что доля людей, живущих выше прожиточного минимума $\beta_{i}$ самая высокая в первой группе, а в третьей и во второй самая низкая : $\beta_{1}>\beta_{2}=\beta_{3}$, индекс дохода $V_{i}$ в первой группе самый высокий и самый низкий в третьей группе: $V_{1}>V_{2}>V_{3}$.

Аналогично, можно объяснить выбор территории 1 мигрирующим населением территории 3.

Из табл. 1 и рис. 2 видно, что снижение подвижности населения территории 2 происходит за счет снижения внутренней миграции и миграции на территорию 3. При этом, миграция на территорию 1 снижается при $q>70$. Снижение активности населения территории 3 так же происходит за счет снижения внутренней миграции и миграции во 

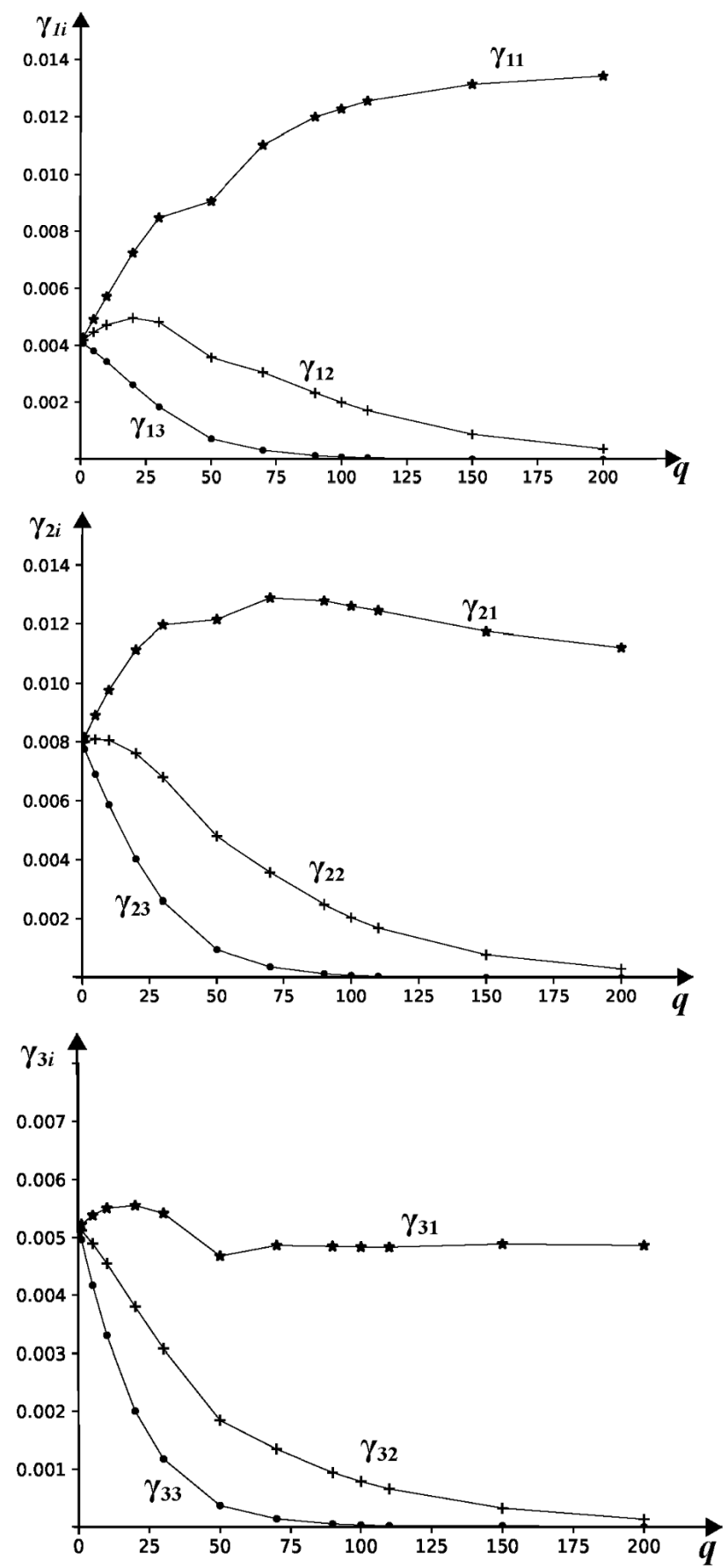

Рис. 2. Миграция из 1, 2 и 3-й группь ФО

[Fig. 2. Migration from the $1^{\text {st }}, 2^{\text {nd }}$, and $3^{\text {rd }}$ groups of the FD]

вторую группу ФО. При этом снижение миграции в первую группу будет происходить при $q \in[20,50]$, а рост при $q \in[0,20] \cup[50,70]$, постоянная доля мигрантов будет наблюдаться при $q \in[70,200]$. Подвижность населения первой группы ФО с ростом $q$ убывает очень медленно, за счет падения внешней миграции и роста внутренней. Из результатов, приве- денных в табл. 1 и на рис. 2, можно сделать вывод, что нестабильность миграции населения в первую группу не способствует росту подвижности населения второй и третьей групп ФО, а рост внутренней миграции с ростом $q$ не способствует росту подвижности населения первой группы ФО.

В реальности рост миграции населения негативно влияет на экономику в целом, поскольку, экономические ресурсы концентрируются в более развитых регионах, а слабые регионы не развиваются из-за оттока населения.

Таким образом, согласно рисункам 1 и 2, для трех групп ФО выполняются следующие условия: $p_{1}>p_{2}>p_{3}$ и $\gamma_{i 1}>\gamma_{i 2}>\gamma_{i 3}, i=1,2,3$, что определяет релевантность рассматриваемых территорий.

Оценим рациональность выбора населения, сравнив модельные результаты со статистическими данными, т. е. реальному процессу будем ставить в соответствие некоторое $q$. На рис. $3 \gamma_{i j}$ - отношение числа мигрирующих из $i$-й группы в $j$-ю к численности населения в $i$-й группы ФО. Для расчета использовались данные из $[17,19]$.

В реальности большая часть мигрирующего населения $i$-й территории мигрирует внутри неё, т. е. $\gamma_{i i}>\gamma_{i j}$ и как показано на рис. 3 не всегда первая группа федеральных округов является наиболее привлекательной для населения второй и третьей группы, а вторая не всегда - для населения первой и третьей. Так из рис. 3 видно, что для населения, покидающего территорию 1 предпочтительнее территория 3, население территории 2 на рассматриваемом промежутке времени колеблется в своем выборе, а для населения покидающего третью группу ФО предпочтительнее первая, чем вторая. Из чего следует, что население наиболее пригодной территории делает наименее рациональный выбор, население второй по релевантности территории колеблется в выборе, а население наименее пригодной территории делает наиболее рациональный выбор.

Это расхождение можно объяснить следующим образом: в реальности существует множество факторов, влияющих на миграцию населения между территориями. В модели учтен 

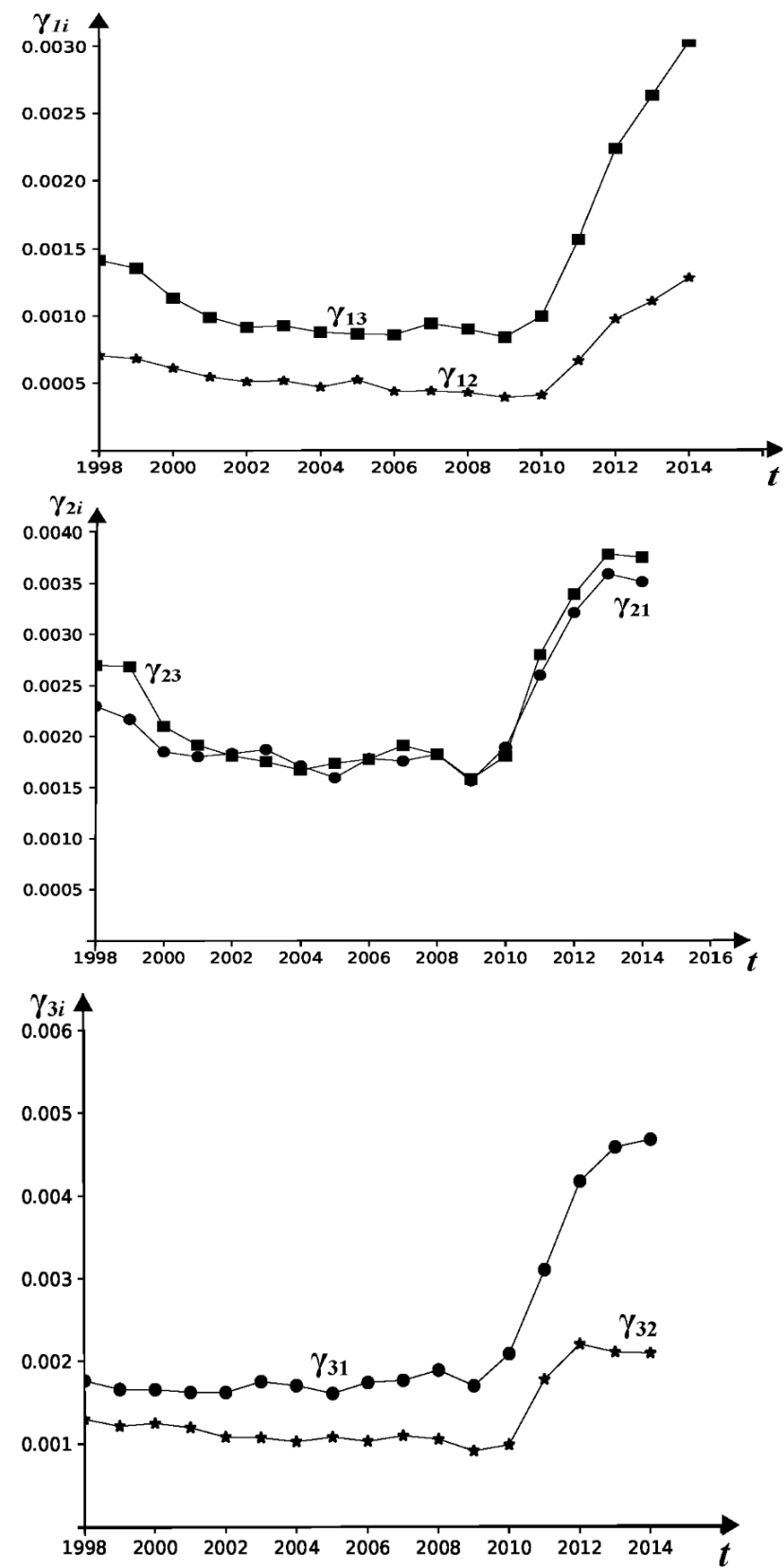

Рис. 3. Реальная миграция из 1, 2 u 3-ŭ групnы $\Phi O$

[Fig. 3. Real migration from the $1^{\text {st, }} 2^{\text {nd }}$, and $3^{\text {rd }}$ groups of the FD]

только один фактор - экономический. При этом с 2010 г. наблюдается скачек миграции населения между территориями, это можно объяснить тем, что в 2008-2010 г. в РФ произошел экономический кризис, что привело к сокращению рабочих мест и отсутствию экономической стабильности в жизни населения, таким образом, трудоспособное население в поисках лучших условий начало усиленно мигрировать. На рис. 4 изображены графики прогноза до 2022 года при значениях $q=0,20,90$.
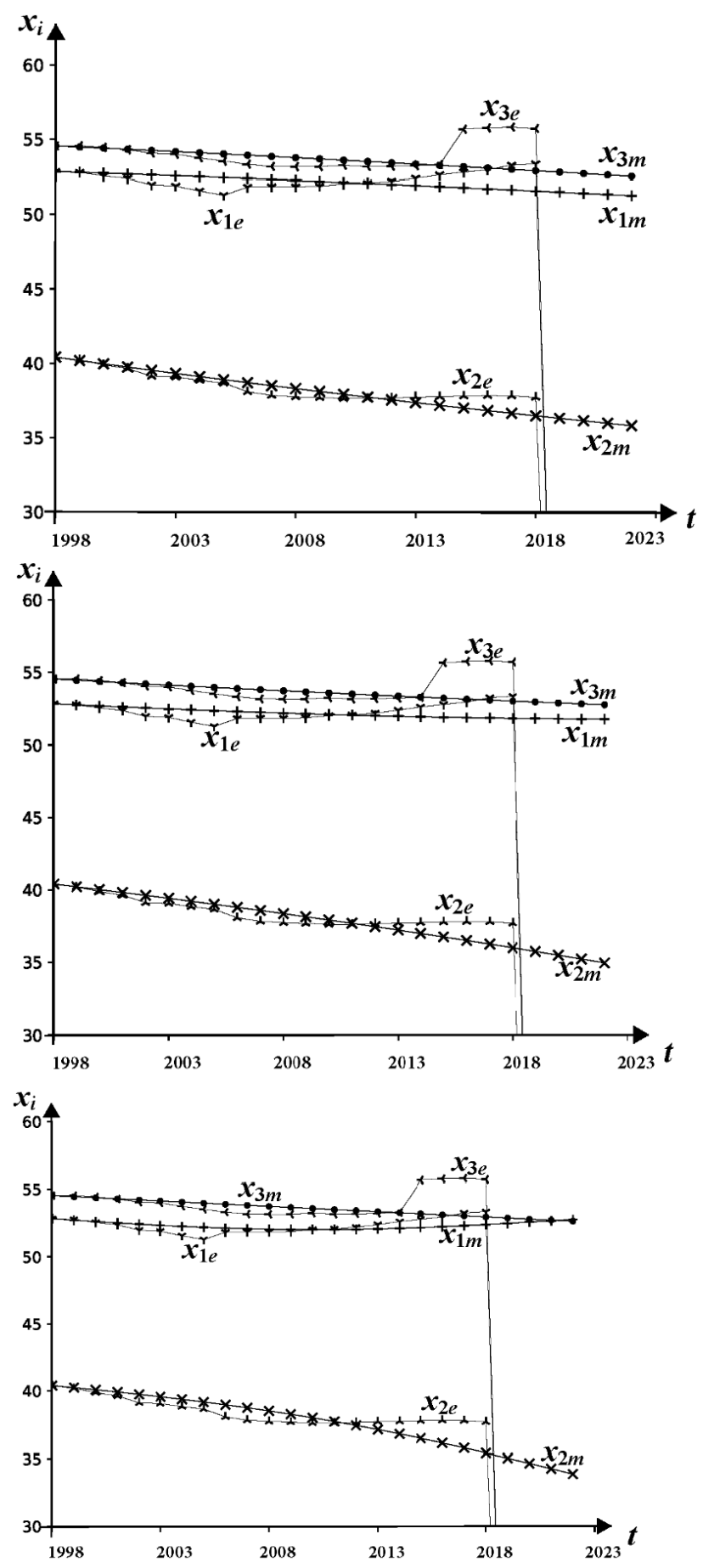

Рис. 4. Прогноз численности населения, при $q=0, q=20, q=90, x_{\text {im }}-$ модельные значения численности для территории $i, x_{i e}$ - реальные значения численности территории $i, x_{i}-$ численность населения территории $i$ млн. чел [Fig. 4. The forecast of the population with $q=0$,

$q=20, q=90, x_{i m}$ are model values of the population for area $i, x_{i e}$ are real values of the population of area $i, x_{i}$ is population of the area $i$ in million people] 


\section{И. В. Данилова, А. Н. Кириллов, А. А. Крижановский}

Из рис. 4 видно, что значение параметра $q$ существенно влияет на прогноз численности населения трех групп ФО. Модель прогнозирует падение численности на территориях 2 и 3 и рост на территории 1 с ростом параметра $q$. При этом, при достаточно малых значениях $q$ модель прогнозирует падение численности на трех территориях ФО. Таким образом, можно сделать вывод, что с ростом параметра оптимальности $q$ рост населения в первой группе ФО будет происходить за счет притока населения, в то время как во второй и третьей группе за счет оттока населения будет происходить падение численности. В третьей группе федеральных округов наблюдается сильное отклонение данных от графика прогноза после 2013 г. Это можно объяснить тем, что присоединение территории Крыма в 2014 г. к территории РФ привело к резкому увеличению численности населения в третьей группе федеральных округов.

В общем случае сложно определить значение $q$ при котором модельные значения численности населения были бы наиболее близким к реальным. Поэтому из рассматриваемых значений параметра $q$ выбирается то, которое удовлетворяет этому условию.

\section{ЗАКЛЮЧЕНИЕ}

Проведена идентификация параметров, характеризующих доли мигрирующего населения и естественного прироста для модели, описывающей динамику численности населения в трех группах федеральных округов РФ с учетом распределения Больцмана. Проведен анализ результатов, полученных на основе идентификации. Проведено сравнение модельных и реальных данных. При этом имеются расхождения между модельными значениями параметров миграционных потоков между тремя территориями РФ и статистическими значениями, это можно объяснить наличием множества факторов, влияющих на миграционные потоки населения, в то время, как в модели рассматривается только один экономический. Построены графики прогноза при некоторых значениях параметра $q$.
Работа выполнена при поддержке РФФИ, грант 18-01-00249a.

\section{КОНФЛИКТ ИНТЕРЕСОВ}

Авторы декларируют отсутствие явных и потенциальных конфликтов интересов, связанных с публикацией настоящей статьи.

\section{СПИСОК ЛИТЕРАТУРЫ}

1. Khavinson, M. Y. Gravitational model of population dynamics / M. Y. Khavinson, M. P. Kulakov // Bulletin SUSU MMCS. - 2017. - Vol. 10, No 3. - P. 80-931. doi: https://doi.org/10.14529/ mmp170307.

2. Василенко, П. В. Гравитационные силы и миграционная подвижность населения региона / П. В. Василенко // Вестник Балтийского федерального университета им. И. Канта. 2013. - Вып. 7. - С. 155-159.

3. Weidlich, W. Sociodynamics: A Systematic Approach to Mathematical Modelling in the Social Sciences / W. Weidlich. - Harwood academic publishers, 2005. $-480 \mathrm{p}$.

4. Charnov, E. L. Optimal foraging, the marginal value theorem / E. L. Charnov // Theoretical Population Biology. - 1976. - Vol. 9. - P. 129 136.

5. The physics of foraging / G. M. Viswanathan [et all]. - Cambridge University Press, 2011. $164 \mathrm{p}$.

6. Кириллов, А. Н. Динамика оптимального поведения двухвидового сообщества с учетом внутривидовой конкуренции и миграции / А. Н. Кириллов, И. В. Данилова // Вестник Удмуртского университета. Математика. Механика. Компьютерные науки. 2019. - Т. 29, вып. 4. - C. 518-531. doi: https:// doi.org/10.20537/vm190404

7. Shuichi, M. Foraging on spatially distributed resources with suboptimal movement, imperfect information, and travelling costs: departures from the ideal free distribution / M. Shuichi, R. Arlinghaus, U. Dieckmann // Oikos. - 2010. - Vol. 119. - P. 1469-1483. doi:10.1111/j.16000706.2010.18196.x

8. Кириллов, А. Н. Динамика распределения популяции по ареалам / А. Н. Кириллов, 
И. В. Данилова // Моделирование и анализ информационных систем. -2018. - Т. 25, № 3. C. 268-275. doi: https://doi.org/10.18255/18181015-2018-3-268-275.

9. Чернавский, Д. С. Синергетика и информация: динамическая теория информации / Д. С. Чернавский. - М. : УРСС, 2016. - 304 с.

10. Balancing selection shapes density - dependent foraging behavior / J. S. Greene [et all] // Nature. - 2016. - № 539. - P. 254-258. doi: 10.1038/nature19848.

11. Calhoun, A. J. The foraging brain / A. J. Calhoun, B. Y. Hayden. // Current Opinion in Behavioral Sciencese. - 2015. - No 5. - P. 24-31. doi: https://doi.org/10.1016/j.cobeha.2015.07.003.

12. Piet, $A$. T. Rats adopt the optimal timescale for evidence integration in a dynamic environment / A. T. Piet, A. El Hady, C. D. Brody // Nature communications. - 2018. - No 9. - P. 42-65. doi:https://doi.org/10.1038/s41467-018-06561-y.

13. Hanks, T. D. Distinct relationships of parietal and prefrontal cortices to evidence accumulation / T. D. Hanks [et all] // Nature. - 2015. - No 520. - P. 220-223. doi:10.1038/nature 14066

14. Evans, D. A. Synaptic threshold mechanism for computing escape decisions / D. A. Evans [et all] // Nature. - 2018. - No 558. - P. 590594. doi:10.1038/s41586-018-0244-6

15. Shenhav, A. Anterior cingulate engagement in a foraging context reflects choice difficulty, not foraging value / A. Shenhav [et all] // Nature neuroscience. - 2014. - No 17. - P. 12491254. doi:10. 1038/nn.3771.
16. Индексы и индикаторы человеческого развития, обновленные данные 2018. - Режим доступа: http://hdr.undp.org/sites/default/ files/2018_human_development_statistical_ update_ru.pdf (Дата обращения: 14.08.2019).

17. Официальный сайт Федеральной службы государственной статистики. - Режим доступа: http://www.gks.ru (Дата обращения: 26.12.2018).

18. United Nations Development Programme Human Development Reports. - Режим доступа: http://hdr.undp.org/en/content/humandevelopment-report-2014 (Дата обращения: 14.08.2019).

19. Официальный сайт Федеральной статистики Росстат. - Режим доступа: https://rosinfostat.ru/vrp (Дата обращения: 26.12.2018).

20. Официальный сайт ЦБ РФ. - Режим доступа: https://cbr.ru (Дата обращения: 26.12.2018).

21. Свидетельство о государственной регистрации программы для ЭВМ № 2020613073, дата государственной регистрации: 10.03.2020. Идентификация параметров в задаче миграции населения / Данилова И. В.; правообладатель Федеральное государственное бюджетное учреждение науки Федеральный исследовательский центр «Карельский научный центр Российской академии наук».

Данилова Инна Владимировна - аспирант лаборатории информационных компьютерных технологий Института прикладных математических исследований Карельского научного центра Российской академии наук. E-mail: DanilovaInna1987@mail.ru

ORCID iD: https://orcid.org/0000-0001-7031-4580

Кириллов Александр Николаевич - д-р физ.-мат. наук, доцент, ведущий научный сотрудник лаборатории информационных компьютерных технологий Института прикладных математических исследований Карельского научного центра Российской академии наук.

E-mail: krllv1812@yandex.ru

ORCID iD: https://orcid.org/0000-0002-3356-1846

Крижановский Андрей Анатольевич - канд. техн. наук, руководитель лаборатории информационных компьютерных технологий Института прикладных математических исследований КарНЦ РАН. E-mail: andrew.krizhanovsky@gmail.com ORCID iD: https://orcid.org/0000-0003-3717-2079 


\title{
BOLTZMANN DISTRIBUTION IN RELATION TO THE PROBLEM OF POPULATION MIGRATION
}

\author{
(C) 2020 I. V. Danilova ${ }^{\bowtie}$, A. N. Kirillov, A. A. Krizhanovsky \\ Institute of Applied Mathematical Research of the Karelian Research Centre \\ of the Russian Academy of Sciences \\ 11, Pushkinskaya Street, 185910 Petrozavodsk, Russian Federation
}

\begin{abstract}
Annotation. In this paper, we consider the problem of migration of the population of the Russian Federation among three groups of federal districts. The first group includes the Central and Northwestern Federal Districts, the second group includes the Far Eastern, Ural, and Siberian Federal Districts, and the third group includes the Southern, Volga, and North Caucasian Federal Districts. A model of population dynamics was proposed taking into account the Boltzmann distribution, which describes the distribution of the migrating population among three specified areas. A utility function included in the Boltzmann distribution was proposed, and a measure of the awareness (attractiveness) of the population in relation to the studied areas was also proposed, which is a part of the utility function. In addition, the measure of awareness takes into account the economic factor, that is the proportion of the population living above the subsistence level and the total population in the considered groups of federal districts. It is assumed that the larger the total population in a given territory, the more information about this territory is available. The parameters characterising the coefficients of natural growth and the proportion of the migrating population of the areas under consideration were identified for various values of the parameter characterising the optimal distribution of the population over the three specified areas. Identification of model parameters was implemented in Python 3.5 using the gradient descent method with step splitting. Also, data from official statistical sources was used to implement the identification of parameters. Based on the identification, the values of the Boltzmann distribution and migration flows among the three groups of federal districts were obtained, the model data was compared with real data, and a population forecast for each area was constructed. It was shown that the value of the parameter of optimality influences the population forecast of all the three groups of federal districts.
\end{abstract}

Keywords: migration, Boltzmann distribution, utility function, awareness measure, identification of parameters.

\section{CONFLICT OF INTEREST}

The authors declare the absence of obvious and potential conflicts of interest related to the publication of this article.

\section{REFERENCES}

1. Calhoun A. J., Hayden B. Y. The foraging brain // Current Opinion in Behavioral Sciencese. 2015. 5. P. 24-31. Available at: doi: https://doi. org/10.1016/j.cobeha.2015.07.003.

Danilova Inna V.

e-mail: DanilovaInna1987@mail.ru
2. Charnov E. L. Optimal foraging, the marginal value theorem // Theoretical Population Biology. 1976. 9, P. 129-136.

3. Chernavskij D. S. Sinergetika i informacyia: dinamicheskaya teoriya informacii [Synergetics and information: dynamic information theory]. Moscow: URSS publ. 2016. (in Russian)

4. Evans D. A., Stempel A. V., Vale R., Ruehle S., Lefler Y., Branco T. A. Synaptic threshold mechanism for computing escape decisions. Nature. 2018 . 558. P. 590-594. Available at: doi: 10.1038/s41586-018-0244-6.

5. Greene J. S., Brown M., Dobosiewicz M., Ishida I. G., Macosko E. Z., Zhang X. Balancing selection shapes density - dependent foraging 
behavior. Nature. 2016. 539. P. 254-258. Available at:doi:10.1038/nature19848.

6. Hanks T. D. Kopec C. D., Brunton B. W., Duan C. A., Erlich J. C., Brody C. D. Distinct relationships of parietal and prefrontal cortices to evidence accumulation // Nature. 2015. 520. P. 220223. Available at: doi:10.1038/nature14066.

7. Khavinson M. Y., Kulakov M. P. Gravitational model of population dynamics. Bulletin SUSU MMCS. 2017. 10(3). P. 80-931. Available at: doi: https://doi.org/10.14529/mmp170307.

8. Kirillov A. N., Danilova I. V. Dinamika raspredeleniya populyacii po arealam [Distribution dynamics of the population by patches]. Modelirovanie i analiz informacionnyh sistem. 2018. 25(3). P. 268-275. Avalable at: doi:https:// doi.org/10.18255/1818-1015-2018-3-268-275.

9. Kirillov A. N., Danilova I. V. Dinamika optimal'nogo povedeniya dvuhvi-dovogo soobshchestva s uchetom vnutrividovoj konkurencii i migracii [Dynamics of the optimal behavior of a two-species community, taking into account intraspecific competition and migration]. Vestnik Udmurtskogo universiteta. Matematika. Mekhanika. Komp'yuternye nauki. 2019. 29(4). P. 518-531. Avalable at: doi: https://doi. org/10.20537/vm190404.

10. Piet A. T., El Hady A., Brody C. D. () Rats adopt the optimal timescale for evidence integration in a dynamic environment. Nature Communications. 2018. 9. P. 42-65. Available at: doi:https://doi.org/10.1038/s41467-018-06561-y.

11. Shenhav A., Straccia M. A., Cohen J. D., Botvinick M. M. Anterior cingulate engagement in a foraging context reflects choice difficulty, not foraging value. Nature. Neuroscience. 2014. 17. P. 1249-1254. Available at: doi:10.1038/nn.3771.

12. Shuichi M., Arlinghaus R., Dieckmann U. Foraging on spatially distributed resources with suboptimal movement, imperfect information, and travelling costs: departures from the ideal free distribution. Oikos. 2010. 119. P. 1469-1483. Available at: doi:10.1111/j.16000706.2010.18196.x.

13. Vasilenko P. V. Gravitacionnije sily b migracionnaja podvizhnost' naselenija regiona. [Gravitational forces and migration mobility of the region's population]. Vestnik Baltijskogo federal'nogo universiteta im. I. Kanta. 2013. 7. P. 155-159. (In Russian).

14. Viswanathan, G. M [et al] The physics of foraging. Cambridge University Press. 2011.

15. Weidlich W. Sociodynamics: A Systematic Approach to Mathematical Modelling in the Social Sciences. Harwood academic publishers. 2005.

16. Human development indices and indicators, updated data. 2018. Available at: http://hdr. undp.org/sites/default/files/2018_human_development_statistical_update_ru.pdf (accessed: 14.08. 2019).

17. The official website of the Federal State Statistics Service. Available at: http://www.gks.ru (accessed: 26. 12. 2018).

18. United Nations Development Programme Human Development Reports. Available at: http:/hdr.undp.org/en/content/human-development-report-2014 (accessed: 14.08.2019).

19. The official website of the Federal Statistics Federal State Statistics Service. Available at: https://rosinfostat.ru/vrp. (accesed: 26.12.2018).

20. The official website of the Central Bank of the Russian Federation. Available at: https://cbr. ru (aaccessed: 26. 12. 2018).

21. Certificate of state registration of a computer program No. 2020613073, date of state registration: 10.03.2020. Identification of parameters in the problem of population migration / Danilova I. V; copyright holder Federal State Budgetary Institution of Science Federal Research Center "Karelian Scientific Center of the Russian Academy of Sciences". 
Danilova Inna V. - graduate student of the Laboratory of Information Computer Technologies, Institute of Applied Mathematical Research, Karelian Scientific Centre of the Russian Academy of Sciences. E-mail: DanilovaInna1987@mail.ru

ORCID iD: https://orcid.org/0000-0001-7031-4580.

Kirillov Alexander N. - Doctor of Physics and Mathematics, Associate Professor, Leading Researcher at the Laboratory of Information Computer Technologies of the Sciences, Institute of Applied Mathematical Research of the Karelian Scientific Centre of the Russian Academy of Sciences.

E-mail: krllv1812@yandex.ru

ORCID iD: https://orcid.org/0000-0002-3356-1846.

Krizhanovsky Andrey A. - PhD in Technical Sciences, Head of the Laboratory of Information Computer Technologies of the Institute for Applied Mathematical Research, Karelian Research Center of the Russian Academy of Sciences.

E-mail: andrew.krizhanovsky@gmail.com

ORCID iD: https://orcid.org/0000-0003-3717-2079 